\title{
Deep SAURON spectral imaging of the diffuse Lyman $\alpha$ halo LAB1 in SSA 22
}

\author{
R. G. Bower, ${ }^{1 \star}$ S. L. Morris, ${ }^{1}$ R. Bacon, ${ }^{2}$ R. J. Wilman, ${ }^{1}$ M. Sullivan, ${ }^{1}$ S. Chapman, ${ }^{3}$ \\ R. L. Davies, ${ }^{4}$ P. T. de Zeeuw ${ }^{5}$ and E. Emsellem ${ }^{2}$ \\ ${ }^{1}$ Institute for Computational Cosmology, University of Durham, South Road, Durham DH1 3LE \\ ${ }^{2}$ CRAL-Observatoire, 9 Avenue Charles-André, 69230 Saint-Genis-Laval, France \\ ${ }^{3}$ California Institute of Technology, MS 320-47, Pasadena, CA 91125, USA \\ ${ }^{4}$ Department of Physics, University of Oxford, Keeble Road, Oxford OX1 3RH \\ ${ }^{5}$ Sterrewacht Leiden, Postbus 9513, 2300 RA Leiden, the Netherlands
}

Accepted 2004 February 27. Received 2004 February 27; in original form 2003 November 6

\begin{abstract}
We have used the SAURON panoramic integral field spectrograph to study the structure of the Ly $\alpha$ emission-line halo, LAB1, surrounding the submillimetre galaxy SMM J221726+0013. This emission-line halo was discovered during a narrow-band imaging survey of the $z=3.1$ large-scale structure in the SSA 22 region. Our observations trace the emission halo out to almost $100 \mathrm{kpc}$ from the submillimetre source and identify two distinct Ly $\alpha$ 'mini-haloes' around the nearby Lyman-break galaxies. The main emission region has a broad line profile, with variations in the line profile seeming chaotic and lacking evidence for a coherent velocity structure. The data also suggest that $\operatorname{Ly} \alpha$ emission is suppressed around the submillimetre source. Interpretation of the line structure needs care because Ly $\alpha$ may be resonantly scattered, leading to complex radiative transfer effects, and we suggest that the suppression in this region arises because of such effects. We compare the structure of the central emission-line halo with local counterparts, and find that the emission-line halo around NGC 1275 in the Perseus cluster may be a good local analogue, although the high-redshift halo is factor of $\sim 100$ more luminous and appears to have higher velocity broadening. Around the Lyman-break galaxy $\mathrm{C} 15$, the emission line is narrower, and a clear shear in the emission wavelength is seen. A plausible explanation for the line profile is that the emission gas is expelled from $\mathrm{C} 15$ in a bipolar outflow, similar to that seen in M82.
\end{abstract}

Key words: galaxies: haloes - galaxies: high redshift.

\section{INTRODUCTION}

A great deal is now known about the properties of star-forming galaxies in the early universe. Measurements of their clustering properties and their luminosity functions has shown that these galaxies are key to understanding the metal enrichment history of the universe (Steidel et al. 1996; Adelberger et al. 2003), while spectroscopic studies have established the role of galactic 'super-winds' in regulating the conversion of baryons into stars (Pettini et al. 1998; Teplitz, McLean \& Becklin 2000; Pettini et al. 2001).

One unexpected outcome of emission-line surveys for starforming galaxies at high redshift has been to establish the existence of large-scale, highly luminous Ly $\alpha$ haloes, termed 'blobs' by Steidel et al. (2000). In this paper, we use the SAURON integral field

^r.g.bower@durham.ac.uk spectrograph to study Ly $\alpha$ blob 1 in SSA 22 (hereafter LAB1). This system is the brighter of two emission haloes that Steidel et al. (2000) discovered during a survey of the conspicuous spike (at $z=3.07-3.11$, Steidel et al. (1998) in the redshift distribution of Lyman-break galaxies in this field. Subsequently, Chapman et al. (2001) discovered the highly obscured, very luminous submillimetre galaxy (SMM J221726+0013) near the centre of this halo. This is possibly a massive elliptical galaxy seen in formation (Eales et al. 1999; Smail et al. 2002).

Using SAURON, we can map the emission-line profiles across the LAB1 structure. This allows us to probe the nature of the ionized gas surrounding the submillimetre source, gaining insight into the origin of the diffuse halo (is it primordial material infalling on to the central object or material expelled during a violent starburst?), the mass of its dark matter halo and the energetics of any super-wind being expelled from the galaxy. We can also trace the large-scale structure surrounding the central source, and investigate 
whether similar haloes surround other galaxies in the field. The answers to these questions will allow us to understand how galaxy formation is regulated in massive galaxies in the high-redshift Universe. They offer key insight into the 'feedback' process and will help explain why less than 10 per cent of the baryon content of the universe ever forms into stars (the 'cosmic cooling crisis', White \& Rees 1978; Cen \& Ostriker 1999; Balogh et al. 2001; Benson et al. 2003).

The gross empirical properties of LAB1 (its spatial extent, luminosity and protocluster environment) are strikingly similar to those of the extended emission-line regions around luminous $z>2$ radio galaxies, but there is no powerful radio jet that could be stirring or ejecting the emitting gas (the radio flux is $<44 \mu \mathrm{Jy}$ at $1.4 \mathrm{GHz}$, Chapman et al. 2001, Chapman et al., private communication). This is an important distinction: the dynamics of the LAB1 halo will give us clearer insight into the confining potential and the nature of the emitting material. Of course, it is likely that the two types of halo are related: several authors (e.g. Willott et al. 2002; Reuland et al. 2003) have suggested that systems like LAB1 are seen during an intense starburst phase that is terminated by the triggering of a powerful radio galaxy.

The layout of the paper is as follows. In Section 2, we describe the observations and the data reduction process. In Section 3, we present a quantitative analysis of the data cube, which we discuss in detail in Section 4. We present our conclusions in Section 5. Throughout, we assume a flat cosmology with $H_{0}=70 \mathrm{~km} \mathrm{~s}^{-1}$ $\mathrm{Mpc}^{-1}, \Omega=0.3$ and $\Lambda=0.7$. This gives an angular scale at $z=3.1 \mathrm{of}$ $7.5 \mathrm{kpc} \operatorname{arcsec}^{-1}$.

\section{THE DATA CUBE}

The SAURON instrument is a high throughput integral field spectrograph (Bacon et al. 2001) that is currently operating on the William Herschel Telescope, La Palma. It was designed and built by a partnership between Lyon, Durham and Leiden with the main objective of studying the dynamics and stellar populations of early-type galaxies (de Zeeuw et al. 2002). It combines wide-field (41 arcsec $\times 33$ arcsec sampled at $0.95 \mathrm{arcsec}$ ) with a relatively high-spectral resolution (4- $\AA$ FWHM, equivalent to $\sigma=100 \mathrm{~km} \mathrm{~s}^{-1}$ in the target rest frame). The instrument achieves this by compromising on the total wavelength coverage, which is limited to the range from 4810 to $5400 \AA$. This spatial and spectral sampling ensure that low-surface brightness features are not swamped by readout noise. However, the limited spectral coverage means that it is only possible to study the Ly $\alpha$ emission from systems at redshifts between $z=2.95$ and 3.45. Fortunately, the SSA 22 supercluster lies within this redshift range. The sky background is devoid of strong night sky emission in the SAURON wavelength range. For these observations, the SAURON grating was upgraded with a volume phase holographic (VPH) grism giving an overall system throughput (including atmosphere, telescope and detector) of 20 per cent. Because the system uses lenslets (rather than fibres) to reformat the focal plane, the field of view is fully tiled and no light is lost between adjacent spectra.

SAURON was used to observe the SSA 22 source for a total of $9 \mathrm{~h}$, spread over three nights in 2002 July. The total integration time was built up from individual 30-min exposures, the telescope being offset by a few arcsecs between each observation. The raw data were reduced using the XSAURON software. The XSAURON software is an adaptation for the SAURON instrument of the public XOASIS software (http://www-obs.univ-lyon1.fr/ oasis/home/index.html). The extraction procedure uses a model for the instrumental distortions to locate each of the spectra, and extracts them using optimal weighting, taking account of the flux overlap between adjacent spectra. An initial sky subtraction step was made using the dedicated sky lenslets of SAURON that sample a blank region 2 arcmin away from the primary field. To remove small flat-field and sky subtraction residuals, a superflat was created using the 18 individual exposures. Dividing by this superflat procedure improved the flat-field accuracy to 1 per cent rms. Each individual data cube was then registered to a common spatial location using the faint star in the south-east of the field and then merged into the final data cube. In the merging process, we choose to set each spectral pixel to a size of 1 arcmin in the spatial dimensions and a size of $1.15 \AA$ in the wavelength dimension. In the combined image, the alignment star has a PSF with 1.5 -arcsec FWHM. Although weak, large-scale residual gradients were evident in the combined frame. To produce the map of Ly $\alpha$ emission, we subtracted this residual continuum, using a low-order polynomial fit to the full wavelength range. This greatly simplifies analysis of the emission-line properties, but means that we cannot attempt to detect any continuum emission associated with the source of Ly $\alpha$ photons. The end result is a three-dimensional (3D; $x, y, \lambda)$ map of the Ly $\alpha$ emission from the region.

\section{RESULTS}

The 3D data produced by SAURON must be carefully visualized to extract the maximum information from the data. We started by creating a colour projection of the data cube shown in Fig 1. In this view, the red, green and blue colour channels have been constructed from the data in the wavelength slices centred on 4964.75, 4976.05 and $4988.70 \AA$. Each channel in the image is $5.6-\AA$ wide $\left(350 \mathrm{~km} \mathrm{~s}^{-1}\right.$ in the system rest frame). We have marked the positions of the

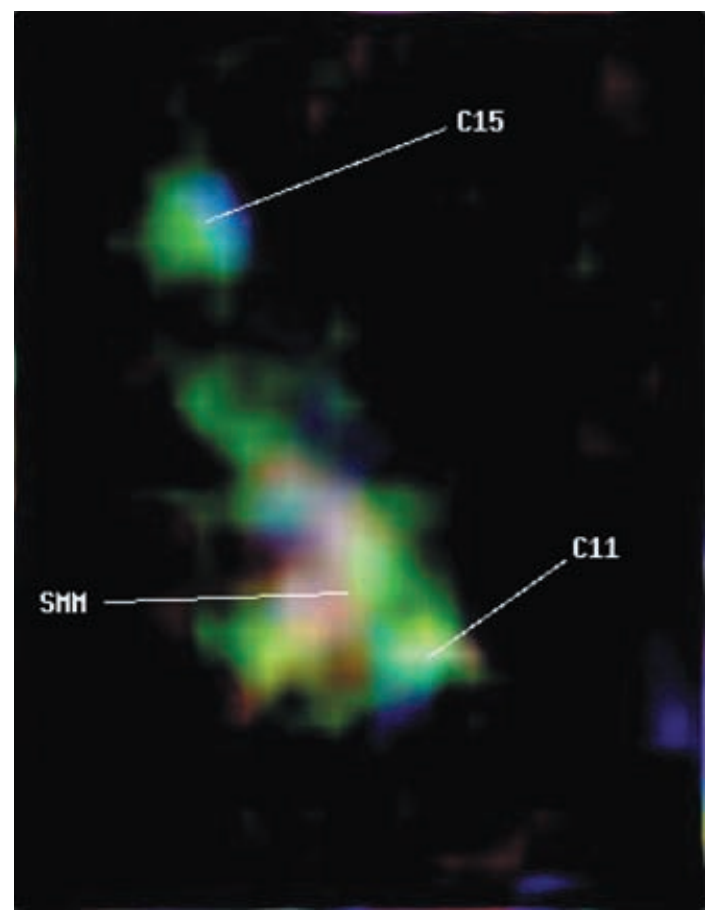

Figure 1. A colour representation of the wavelength distribution of Ly $\alpha$ emission in LAB1. A simple interpretation of the image is that red, green and blue channels represent the redshifted and blueshifted motions of the ionized material in the halo. The positions of the two Lyman-break galaxies $\mathrm{C} 11$ and $\mathrm{C} 15$ are marked, along the position of the submillimetre source (SMM). The area shown is $33 \operatorname{arcsec} \times 42 \operatorname{arcsec}$. 

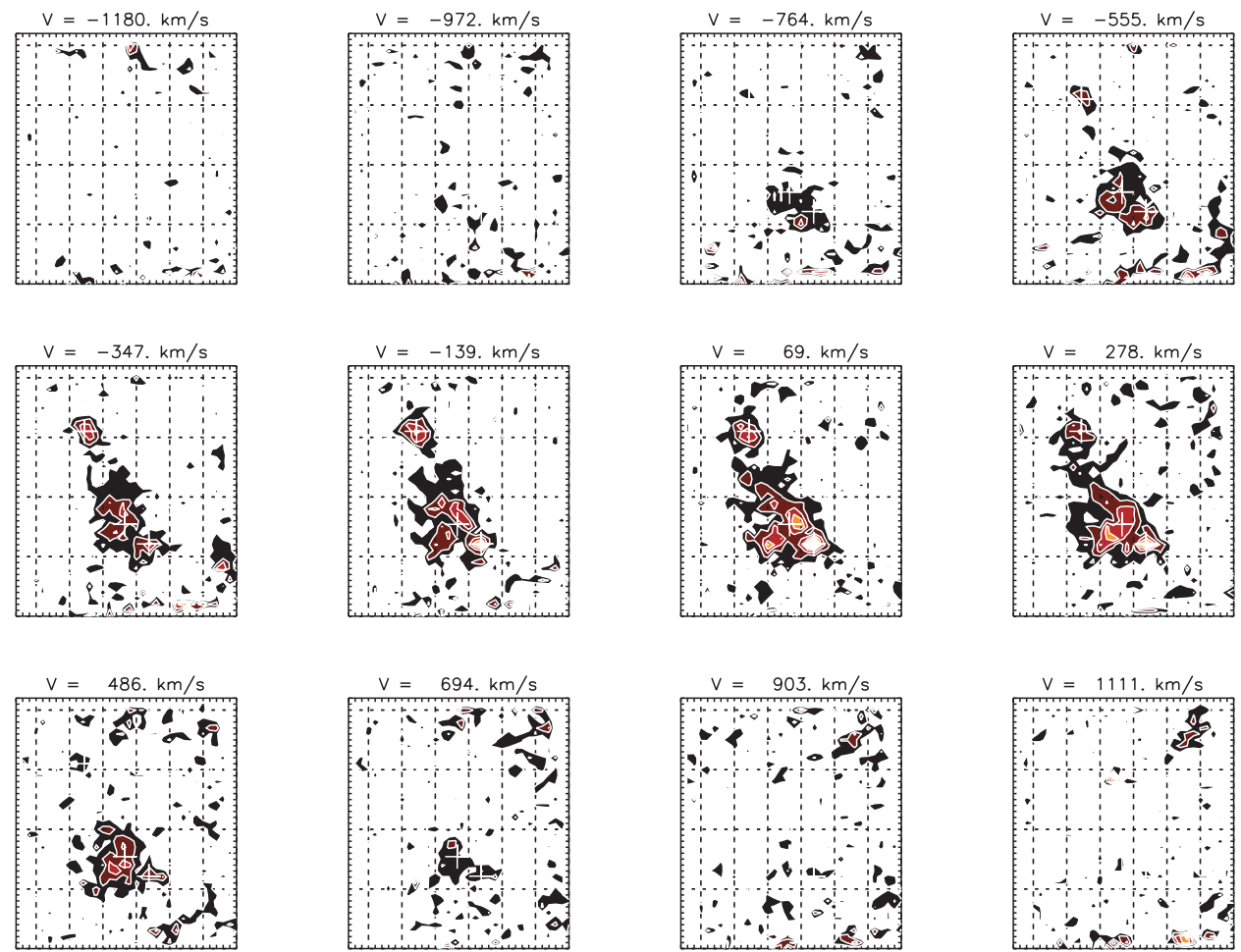

Figure 2. A sequence of contour plots showing the changing morphology of the Ly $\alpha$ emission at different wavelengths. The velocity step between each map is $208 \mathrm{~km} \mathrm{~s}^{-1}$, with each slice combining a 5.6- $\AA$ wavelength range so that alternate panels show independent data. Zero velocity corresponds to a Ly $\alpha$ redshift of 3.10. Crosses mark the positions of Lyman-break galaxies and the submillimetre source. The grid squares have a spacing of 10 arcsec. The faintest contour has a surface brightness of $8.6 \times 10^{-19} \mathrm{erg} \mathrm{s}^{-1} \mathrm{~cm}^{-2} \operatorname{arcsec}^{-2}$.

Lyman-break galaxies, C11 and C15, identified by Steidel et al. $(1996,2000)$ and the location of the submillimetre source identified by Chapman et al. (private communication; see below). The data cube can alternatively be viewed as a sequence of wavelength slices as shown in Fig 2, or these slices can be combined together to make an animation (this can be viewed at http://starwww.dur.ac.uk/ $\sim$ rgb/SSA 22 movie.mpg).

Many striking structures can be clearly seen in the main halo. The overall width of the emission line is very broad $\left(\sim 500 \mathrm{~km} \mathrm{~s}^{-1}\right.$ FWHM) but separate emission structures can be identified. There is significant velocity shear in the emission region around the Lymanbreak galaxies $\mathrm{C} 11$ and $\mathrm{C} 15$, while the structure across the main halo seems more chaotic. The morphology of the diffuse emission can be seen in these velocity slices: particularly interesting is the depression seen near the centre of the halo (this is partly filled by redshifted emission), and the diffuse extension of the halo towards the nearby Lyman-break galaxy C15. C15 itself is centred in a distinct but much smaller halo. We discuss each of these features next.

To further quantify the emission and its spatial variations, we fitted each spectrum with a single Gaussian line of variable position, width and normalization. The best-fitting parameters for each lenslet are shown in Fig 3, while some example spectra and their associated fits are shown in Fig 4. The limiting surface brightness at which we are able to reliably detect and fit to the line is $2 \times 10^{-18}$ erg $\mathrm{s}^{-1} \mathrm{~cm}^{-2} \operatorname{arcsec}^{-2}$ for lines with $\sigma=5 \AA$. Clearly, fitting a single Gaussian to the emission may not correctly represent the underlying flux distribution. For this reason, the line-fitting approach and the wavelength slices discussed earlier should be viewed as complementary rather than as alternatives.

If we concentrate on the emission in a 6-arcsec diameter circle around the central SCUBA source, as shown in the figure, the average velocity width $(\sigma)$ is $560 \mathrm{~km} \mathrm{~s}^{-1}$. The shifts in the central wavelength are much smaller than this: we get an rms lenslet to lenslet variation of only $80 \mathrm{~km} \mathrm{~s}^{-1}$. Around $\mathrm{C} 11$, the line has similar average width $\left(470 \mathrm{~km} \mathrm{~s}^{-1}\right)$, but the rms velocity shift between lenslets is much larger $\left(240 \mathrm{~km} \mathrm{~s}^{-1}\right)$. The total flux from the complete region (including $\mathrm{C} 11$ ) that is detected by the line-fitting algorithm is $1.7 \times 10^{-15} \mathrm{erg} \mathrm{s}^{-1} \mathrm{~cm}^{-2}$ (in good agreement with Steidel et al. 2000), corresponding to a luminosity of $1.4 \times 10^{44} \mathrm{erg}$ $\mathrm{s}^{-1}$ in our adopted cosmology.

In the emission region around $\mathrm{C} 15$, the line is somewhat narrower $\left(360 \mathrm{~km} \mathrm{~s}^{-1}\right)$, and the rms shift in central velocity $\left(110 \mathrm{~km} \mathrm{~s}^{-1}\right)$, which is dominated by the velocity asymmetry that is prominent in the wavelength slices. The flux from a 6 -arcsec diameter region centred on $\mathrm{C} 15$ is $1.3 \times 10^{-16} \mathrm{erg} \mathrm{s}^{-1} \mathrm{~cm}^{-2}$.

The optical counterpart of the submillimetre source has been identified by (Chapman et al., 2003; see also Ohyama et al. 2003) after detecting the associated $\mathrm{CO}$ emission. To locate the emission relative to the submillimetre source more precisely, we aligned the data cube and the Hubble Space Telescope (HST) image of Chapman et al. using the positions of the alignment star and the Lyman-break galaxies $\mathrm{C} 11$ and $\mathrm{C} 15$. Fig 5 shows the STIS image overlayed with the contours of the total Ly $\alpha$ emission. This clearly shows the location of the submillimetre source close to the centre of the 'cavity' in the emission structure.

\section{DISCUSSION}

Below we divide our results into the separate features seen in our data and discuss some ideas for how we might interpret them. The interpretation is complicated because $\operatorname{Ly} \alpha$ is a resonant line. Thus 

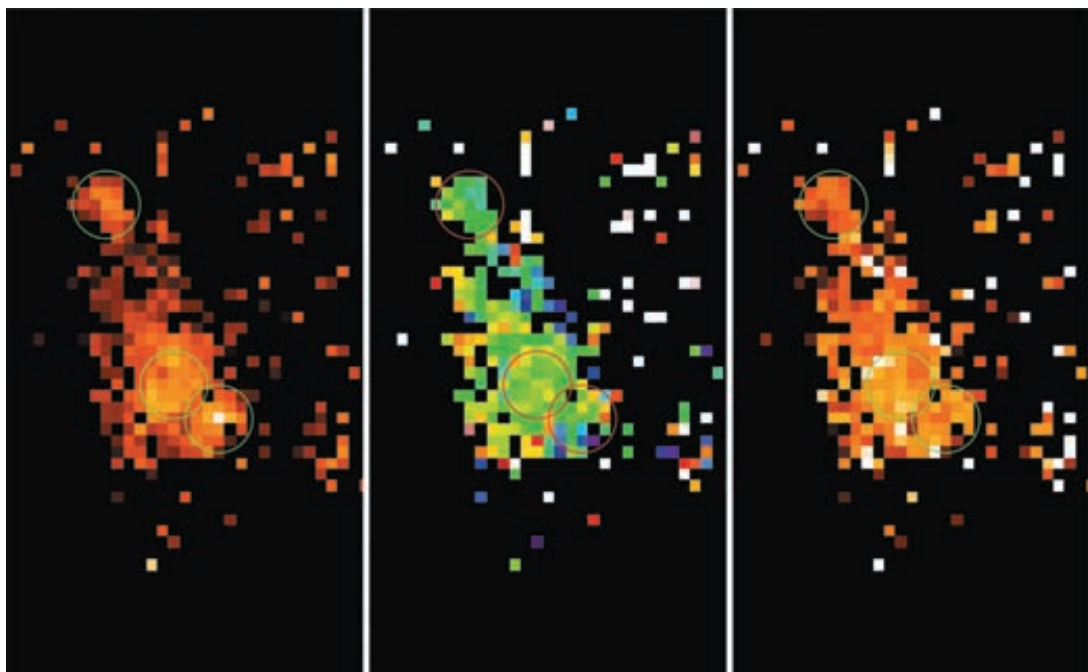

Figure 3. Single Gaussian fits to the data. Left-hand panel, the intensity of the fitted line (red-white: $0-2 \times 10^{-17} \mathrm{erg} \mathrm{s}^{-1} \mathrm{~cm}^{-2} \mathrm{arcsec}^{-2}$ ). Middle panel, the central wavelength of the line (blue-red: $4970-4990 \AA$ ). Right-hand panel, the width of the line (red-white: $\sigma=0-15 \AA$ ). The plots allow us to quantify the velocity structure seen in the halo. The circles show the regions used to compute the average line width and variation in the line centroid. Examples of the line fits are shown in Fig 4.

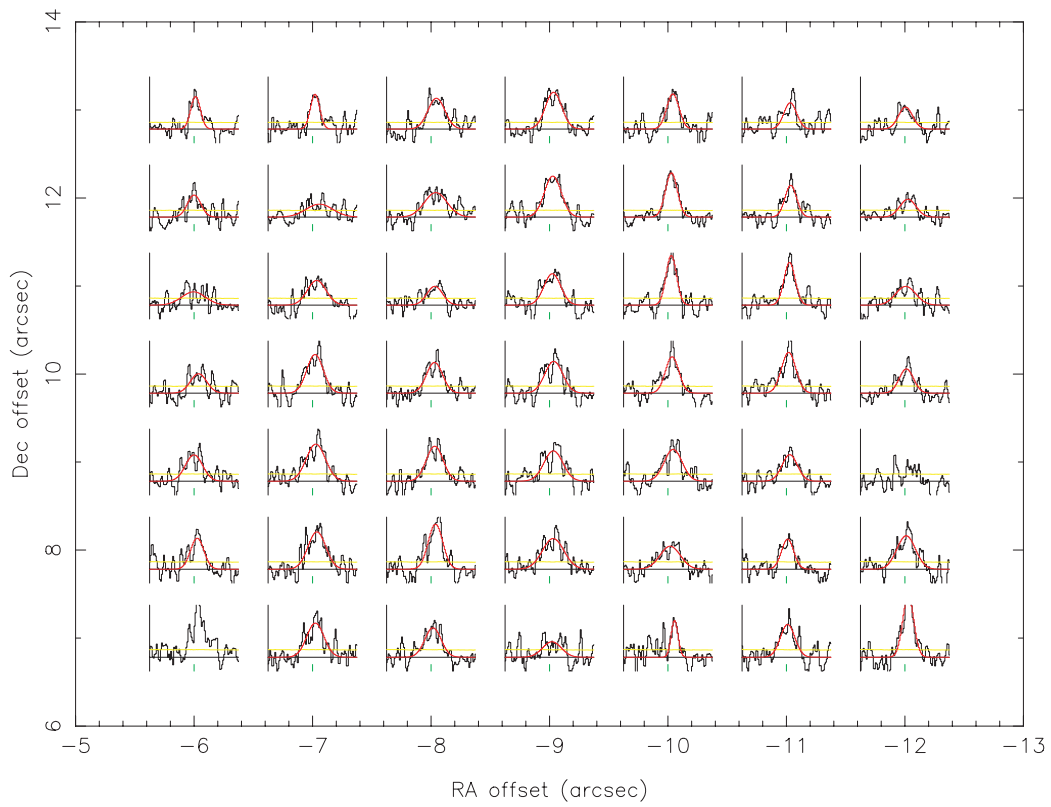

Figure 4. The SAURON spectra (black) and the best-fitting Gaussian model (red), for emission in the region surrounding SMM J221726+0013. The spectra cover 4930-5030 ̊ in the observed frame (equivalent to $\pm 3000 \mathrm{~km} \mathrm{~s}^{-1}$ ). The green tick marks $4980 \AA$. The parameters of the line fits are shown in Fig 3 . The yellow line shows an estimate of the $1 \sigma$ noise level. In this coordinate system SMM J221726+0013 is roughly centred on $(-9,10)$ while C11 is centred on $(-13,7)$.

shifts in the feature can appear because of both genuine gas motion and photon diffusion in wavelengths to escape from optically thick regions: in regions where the ionized halo is dense and the gas bulk velocity is relatively constant, Ly $\alpha$ photons must diffuse further into the wings of the line to escape. We thus expect the changes in the profile to reflect both the bulk motion of the material and the variation of optical depth and dust obscuration with radius (e.g. Meinköhn \& Richling 2002). In what follows we assume that bulk or turbulent motion is the dominant source of line broadening. This seems to be an adequate approximation. Meinkoln \& Richling show that in static media, the line becomes clearly double peaked before the resonant scattering can significantly boost the FWHM linewidth. Their simulations nevertheless show the inherent difficultly of interpreting the profiles of resonantly scattered emission lines.

\subsection{The velocity structure of LAB1}

The main halo has a complex structure. Within the broad emission, there are many halo components. The variations in linewidth and velocity are inconsistent with a simple outflowing thin shell. The distribution is better modelled as many distinct gas components, moving relative to each other with speeds of several hundred $\mathrm{km} \mathrm{s}^{-1}$. 
One (certainly naive) interpretation of the wavelength variations is that they reflect the motions of separate gas clumps bound in a common gravitational potential.

If the above were true, we could use the magnitude of the velocity width to infer the halo mass within $\sim 75 \mathrm{kpc}$ (or 10 arcsec, the typical radius at which the emission can be fitted). If we assume that the clumps are on random orbits with a line-of-sight velocity dispersion of $500 \mathrm{~km} \mathrm{~s}^{-1}$, the virial theorem suggests a mass of order $1.3 \times$ $10^{13} \mathrm{M}_{\odot}$, as expected for a small cluster with a density distribution given by the Navarro, Frenk \& White (1997) parametrization. It is likely, however, that in fact the clumps have a net outflow or inflow, or are subject to drag from the intergalactic medium (IGM). This makes the mass estimate highly uncertain.

It is interesting to compare the Ly $\alpha$ emission morphology to the $\mathrm{H} \alpha$ emission filaments seen in some local clusters such as Perseus. The H $\alpha$ seen around NGC 1275, the central galaxy of the Perseus cluster, can be traced over a radial extent of $70 \mathrm{kpc}$ (Conselice, Gallagher \& Wyse 2001). This is comparable to the extent of the emission region around SMM J221726+0013. Diffuse Ly $\alpha$ was indeed detected around NGC 1275 with the International Ultraviolet Explorer satellite (Fabian et al. 1984). Fabian et al., estimate a total Ly $\alpha$ luminosity of $3 \times 10^{42} \mathrm{erg} \mathrm{s}^{-1}$ based on the assumption that the emission region has comparable extent to the $\mathrm{H} \alpha$ line filaments. This is a factor one hundred time smaller than the luminosity of LAB1. Concelise et al. also measure the spread of velocities of the NGC 1275 filaments (over a 14-kpc region). They find a generally chaotic pattern, albeit with weak evidence for rotation. The bulk velocity is comparable to the rms spread in the line centres (both are $\sim 150 \mathrm{~km} \mathrm{~s}^{-1}$ ) but the velocity scale is slower than we find around SMM J221726+0013 (although we recognize that caution is required as we have ignored the role of resonant scattering). Although the luminosity and flow velocities around the NGC 1275 system are weaker, it may still be a good local analogue for the SMM J221726+0013 system, and may give us insight into the physical origin of the higher redshift halo. Conselice et al. conclude that the emission is driven by the interaction of outflowing material (in the form of hot bubbles rising under their buoyancy) and the inflow of material cooling in the cluster potential (see also Fabian et al. 2003; Bruggen \& Kaiser 2003).

In the high-redshift case, cooling alone is unlikely to explain the observed Ly $\alpha$ emission, because the Ly $\alpha$ luminosity is so high compared to the limits on the X-ray emission $\left(L_{X} / L_{\mathrm{Ly} \alpha}<10\right.$, Chapman et al., private communication); in a intracluster medium cooling from a few kiloelectronvolts, we would expect $L_{X} / L_{\mathrm{Ly} \alpha}>10^{3}$ even if each hydrogen atom emits a Ly $\alpha$ photon (Cowie, Fabian \& Nulsen 1980). However, at such high redshift it is far from clear that the gas is ever heated to the virial temperature of the system. The simulations of Fardal et al. (2001) suggest that cooling is so rapid that a hydrostatic equilibrium is never established. In their interpretation, both the extent of the emission halo and the width of the emission line are due to radiative transfer effects. This seems unlikely since the linewidth appears to increase with radius (rather than decline), but much more detailed radiative transfer models are needed to test this hypothesis. Our preferred explanation is that the extent and distribution of line flux reflect the underlying motion and extent of cooling gas. A powerful collimated outflow appears inconsistent with the lack of velocity shear across the halo (unless it is oriented close to the line of sight). The emission halo therefore seems best explained by the interaction of slowly rising buoyant material with cooling gas contained in the protocluster potential. The origin of this hot material remains unclear, however: the convection could equally

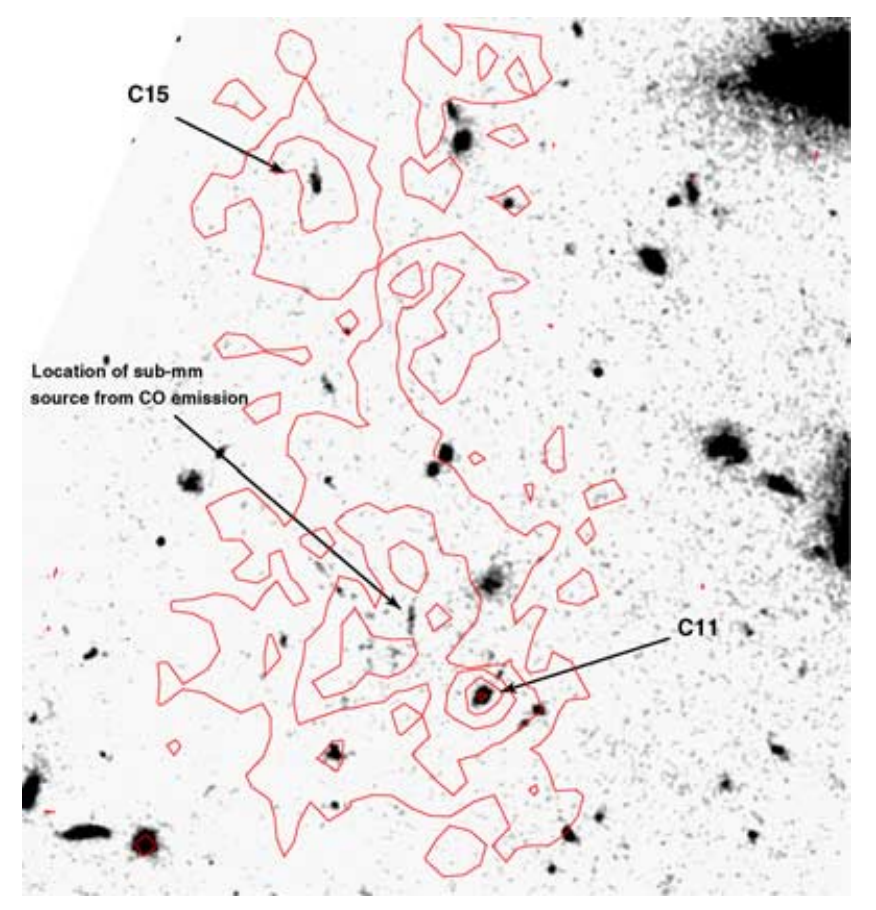

Figure 5. A deep HST image of the SSA 22 LAB1 region showing the position for the submillimetre counterpart relative to the total Ly $\alpha$ emission (contours). The submillimetre source may lie in a 3D cavity in the emission (compare contours with Fig 1). The Lyman-break galaxies C15 and C11 are marked: their distinct haloes are clearly seen in the 3D data set.

well be driven by an heavily obscured active galactic nucleus (AGN) or by vigorous star formation.

\subsection{Relationship to the submillimetre source}

Fig 5 shows the relative location of the emission-line halo and the optical counterpart of the strong submillimetre source (Chapman et al. 2001, Chapman et al., private communication). The overlay suggests that the submillimetre source may be located at the centre of a 'cavity' in the Ly $\alpha$ emission. It has already been established that the Ly $\alpha$-emission haloes in Perseus (Johnstone \& Fabian 1988) and high-redshift radio galaxies (van Breugel et al. 2002) are ionized by a distributed source. The presence of the cavity supports this interpretation - it is hard (although not impossible) to construct emission geometries in which the central source ionizes the surrounding nebulae but is itself shielded from the observer.

There are several possible interpretations of the cavity.

(i) It may be a genuine cavity in the ionized gas distribution. This might be evidence for a strong wind blowing away from the central submillimetre source. However, we would then expect the broadest lines to be observed close to the centre. This is in conflict with the situation observed.

(ii) Fig 5 could also suggest that we are seeing an effect similar to that responsible for wide angle tail radio sources. The relative locations of the HST continuum object compared to the contours of the Ly $\alpha$ emission could be consistent with an object blowing out line emitting gas that is then slowed by ram pressure, losing collimation in the process and hence producing two large trailing lobes of emission. The physical details of the emission process are clearly completely different, but the slowing of the gas by the intercluster medium could be similar. It is unclear, however, why the 
material would remain ionized (given its very high luminosity) for a sufficiently long period of time.

(iii) Alternatively, the cavity may occur because the ionized gas in this region contains significant dust (cf. Reuland et al. 2003). As the Ly $\alpha$ diffuses out of the central region, it is strongly extincted. This explanation is appealing since we know that the central SCUBA source has high extinction. If there were no dust at all, we would expect the emission to be scattered into the line wings rather than being absorbed. This scattering may be why the emission that is seen near the location of the submillimetre source tends to be redshifted.

\subsection{Mini-haloes around Lyman-break galaxies}

The two other Lyman-break galaxies embedded in the structure appear to have dynamically distinct haloes. This is particularly clear for $\mathrm{C} 15$ to the north of the main halo (although there is faint emission that bridges between $\mathrm{C} 15$ and the central halo). A similar halo can also be discerned around $\mathrm{C} 11$. This is a surprising discovery that leads us to speculate that other Lyman-break galaxies would also have extended Ly $\alpha$ haloes if observed to sufficiently low-surface brightness. The mini-halo around the $\mathrm{C} 15$ Lyman-break galaxy has its own characteristic velocity shear pattern. We can identify the morphology of this galaxy from the HST imaging of Chapman et al. (2003). C15 is elongated at roughly $60^{\circ}$ (Fig 5) to the velocity shear seen in Ly $\alpha$; interestingly, the morphology of C15 seems somewhat more disturbed than typical Lyman-break galaxies. The orientation, together with the unflattened morphology of the emission, makes it unlikely that the shear reflects the rotation of a conventional gas disc. Instead, the shear pattern is reminiscent of the super-wind outflows predicted from protogalactic discs (Springel \& Hernquist 2003), and observed in local starburst galaxies such as M82.

If we interpret the shear as an outflow inclined at $\sim 45^{\circ}$ to the line of sight, the pattern suggests a deprojected outflow velocity of $\sim 200 \mathrm{~km} \mathrm{~s}^{-1}$, and a physical extent of $\sim 40 \mathrm{kpc}$. For comparison, the $\mathrm{H} \alpha$ emission seen in M82 indicates a deprojected outflow velocity of $600 \mathrm{~km} \mathrm{~s}^{-1}$ (Shopbell \& Bland-Hawthorn 1998), extending to $11 \mathrm{kpc}$ above the disc (Devine \& Bally 1999). It is interesting that the dwarf galaxy M82 has a significantly larger outflow velocity than $\mathrm{C} 15$ even though Lyman-break galaxies are thought to have high disc circular velocities ( $200 \mathrm{~km} \mathrm{~s}^{-1}$, Pettini et al. 1998).

However, the geometry of the flow in $\mathrm{C} 15$ is qualitatively different, and does not share the same narrow cone shaped structure as M82 and other local starburst galaxies (as far as can be discerned from our data). It is tempting to suggest that star formation is sufficiently vigorous that the wind is being driven from across the whole galaxy disc, rather than just by the nuclear starburst as seen in M82; but examination of the observed morphology of C15 does not suggest a simple disc galaxy, and so a direct morphological comparison is, perhaps, inappropriate.

\section{CONCLUSIONS}

We have used the SAURON panoramic integral field spectrograph to study the structure of the Ly $\alpha$ emission-line halo surrounding the submillimetre galaxy SMM J221726+0013. The emission halo can be traced out to almost $100 \mathrm{kpc}$ from the submillimetre source, and the two nearby Lyman-break galaxies are shown to have kinematically distinct emission-line haloes of their own. The main features that we can discern are as follows. (i) The emission-line profile around the central submillimetre source is broad, $\sigma \sim 9 \AA$. While the line profile varies significantly around the submillimetre source, there is no coherent variation in the line centroid.

(ii) Ly $\alpha$ emission appears suppressed in the immediate vicinity of the submillimetre source.

(iii) The Lyman-break galaxies C15 and C11 appear to be associated with enhancements in the emission. These 'mini-haloes' show significant velocity shear.

If we interpret the broad width of the emission line as being due to velocity motion of individual gas clouds, we infer line-of-sight velocities of $\sim 500 \mathrm{~km} \mathrm{~s}^{-1}$, suggesting a dark halo mass of $1.3 \times$ $10^{13} \mathrm{M}_{\odot}$ as expected for a small cluster. We compare the emission halo to the emission filaments surrounding NGC 1275, the central galaxy of the Perseus cluster. The chaotic velocity structure and the extent of the emission are similar, although the Ly $\alpha$ luminosity of LAB1 is two orders of magnitude larger. Combined with the lack of coherent velocity shear and the high ratio of the Ly $\alpha$ and X-ray flux, the comparison leads us to speculate that the emission halo of SMM J221726+0013 is powered by the interaction between cooling gas and a relatively weak outflow from the central source. Our data do not distinguish whether this flow is driven by vigorous star formation or by a heavily obscured AGN. It is clear, however, this interpretation needs to be confirmed by combining radiative transfer models with realistic simulations of massive galaxy formation in the early universe.

The structure of emission halo suggests a cavity around SMM J221726+0013. While one possible explanation is that this region has been filled with hot, completely ionized material, the dip in the emission may equally be explained because of dust obscuration in the material ejected from the submillimetre source.

The 'mini-haloes' around the two Lyman-break galaxies in the field (C11 and C15) show clear velocity shear across their emission haloes. The structure appears to be consistent with a bipolar outflow of material, similar to that seen in the starbursting dwarf galaxy M82. If the material is an outflow, the deprojected velocity of the flow is $\sim 200 \mathrm{~km} \mathrm{~s}^{-1}$, less than the velocity inferred for the outflow from M82, and less than the outflow velocities inferred by Pettini et al. (1998, see also Teplitz et al. 2000; Pettini et al. 2000, 2001) from comparison of the redshifts of Ly $\alpha$ and nebular emission lines in the rest-frame optical.

These observations break new ground for the SAURON instrument. Although it was designed to study the dynamics and stellar populations of nearby elliptical galaxies, we have shown that it can very effectively be used to study low surface brightness emission features only detectable in long integrations. It is interesting to speculate how far this powerful technique can be taken. On the one hand it is key to establish whether the diversity of structure seen in SSA 22 LAB1 is a generic property of other highly luminous submillimetre galaxies, or whether the deep potential well of the SSA 22 supercluster is necessary to produce emission of this luminosity and extent. It will also be important to determine whether other Lyman-break galaxies show mini-haloes similar to C15 (cf. Fynbo et al. 2002; Matsuda et al. 2004). The observations that we have presented provide a foretaste of the deeper observations that will be made with integral field spectrographs on 8-m telescopes such as GMOS (Davies et al. 1997), CIRPASS (Parry et al. 2000) and SPIFFI (Eisenhauer et al. 2000, see Bunker et al. 2004, for a recent review). Of particular interest is the combination of large telescope aperture and relatively wide field that is offered by VIMOS (Le Fevre et al. 2000) and the planned MUSE integral field spectrograph (Henault et al. 2003). 
These instruments will open the way for deep blank-field surveys of emission-line galaxies in the high-redshift universe.

\section{ACKNOWLEDGMENTS}

We thank the SAURON instrument team for their support of this programme, and for creating an instrument with the superb sensitivity of SAURON. It is a pleasure to thank the ING staff, in particular Rene Rutten, Tom Gregory and Chris Benn, for enthusiastic and competent support on La Palma. The construction of SAURON was made possible through grants 614.13.003 and 781.74.203 from ASTRON/NWO and financial contributions from the Institut National des Sciences de l'Univers, the Université Claude Bernard Lyon-I, the universities of Durham and Leiden, the British Council, PPARC grant 'Extragalactic Astronomy \& Cosmology at Durham 19982002' and the Netherlands Research School for Astronomy NOVA. RGB is pleased to acknowledge the support of fellowships from PPARC and the Leverhulme foundation.

\section{REFERENCES}

Adelberger K., Steidel C., Shapley A. E., Pettini M., 2003, ApJ, 584, 45 Bacon R. et al., 2001, MNRAS, 326, 23

Balogh M. L., Pearce F. R., Bower R. G., Kay S., 2001, MNRAS, 326, 1228

Benson A. J., Bower R. G., Frenk C. S., Lacey C. G., Baugh C. M., Cole S., 2003, ApJ, 599, 38

Bruggen M., Kaiser C., 2003, Nat, 418, 301

Bunker A. J. et al., 2004, Astr. Nach., 325, 139

Cen R., Ostriker J. P., 1999, ApJ, 514, 1

Chapman S., Lewis G., Scott D., Richards E., Borys C., Steidel C. C., Adelberger K. L., Shapley A. E., 2001, ApJ, 548, 17

Chapman S. C., Windhorst R., Odewhan S., Yan H., Conselice C., 2003, ApJ, 599, 92

Conselice C. J., Gallagher J. S., Wyse R. F. G., 2001, AJ, 122, 2281

Cowie L. L., Fabian A. C., Nulsen P. E. J., 1980, MNRAS, 191, 399

Davies R. L. et al., 1997, SPIE, 2871, 1099

de Zeeuw T. et al., 2002, MNRAS, 329, 513

Devine D., Bally J., 1999, ApJ, 510, 197
Eales S., Lilly S., Gear W., Dunne L., Bond J. R., Hammer F., Le Fèvre O., Crampton D., 1999, ApJ, 515, 518

Eisenhauer F., Tecza M., Mengel S., Thatte N. A., Roehrle C., Bickert K., Schreiber J., 2000, SPIE, 4008, 289

Fabian A. C., Sanders J. S., Crawford C. S., Conselice C. J., Gallagher J. S., Wyse R. F. G., 2003, MNRAS, 344, L48

Fabian A. C., Nulsen P. E. J., Arnaud K. A., 1984, MNRAS, 208, 179

Fardal M. A., Katz N., Gardner J. P., Hernquist L., Weinberg D. H., Davé R., 2001, ApJ, 562, 605

Fynbo J. P. U. et al., 2002, A\&A, 388, 425

Henault F. et al., 2003, SPIE, 4841, 1096

Johnstone R. M., Fabian A. C., 1988, MNRAS, 233, 581

Le Fevre O. et al. 2000, 2000, SPIE, 3355, 8

Matsuda Y. et al., 2004, ApJ, in press

Meinköhn E., Richling S., 2002, A\&A, 392, 827

Navarro J. F., Frenk C. S., White S. D. M., 1997, ApJ, 490, 493

Ohyama Y. et al., 2003, ApJ, 591, L9

Parry I. R. et al., 2000, SPIE, 4008, 1193

Pettini M., Kellog M., Steidel C. S., Dickinson M., Adelberger K., Giavalisco M., 1998, 508, 539

Pettini M., Steidel C. S., Adelberger K., Dickinson M., Giavalisco M., 2000, ApJ, 528, 96

Pettini M., Shapley A. E., Steidel C. C., Cuby J. -G., Dickinson M., Moorwood A. F. M., Adelberger K., Giavalisco M., ApJ, 554, 981

Reuland M. et al., 2003, ApJ, 592, 755

Shopbell P. L., Bland-Hawthorn J., 1998, ApJ, 493, 129

Smail I., Ivison R., Blain W. A., Kneib J.-P., 2002, MNRAS, 331, 495

Springel V., Hernquist L., 2003, 339, 312

Steidel C. C., Giavalisco M., Dickinson M., 1996, ApJ, 462, L17

Steidel C. C., Adelberger K. L., Dickinson M., Giavalisco M., Pettini M., Kellogg M., 1998, ApJ, 492, 428

Steidel C. C., Adelberger K. L., Shapley A. C., Pettini M., Dickinson M., 2000, ApJ, 532, 170

Teplitz H. I., McLean I. S., Becklin E. E., 2000, ApJ, 533, L63

van Breugel W. et al., 2002, preprint (astro-ph/0209173)

White S. D. M., Rees M. J., 1978, MNRAS, 183, 341

Willott C. J., Rawlings S., Archibald E. N., Dunlop J. S., 2002, MNRAS, 331,435

This paper has been typeset from a $\mathrm{T}_{\mathrm{E}} \mathrm{X} / \mathrm{L}_{\mathrm{E}} \mathrm{T}$ file prepared by the author. 